\title{
Resiliencia y Apoyo Social como Predictores del Duelo Perinatal en Mujeres Mexicanas: Modelo Explicativo
}

\author{
Resilience and Social Support as Predictors of Perinatal Grief in Mexican Women: \\ Explanatory Model
}

\author{
Cecilia Mota González ${ }^{1}$, Nazira Calleja², Claudia Sánchez Bravo ${ }^{3}$, Jorge Carreño Meléndez ${ }^{4}$ y \\ Jessica L. Balbuena Buendía ${ }^{5}$
}

\begin{abstract}
Resumen
La pérdida perinatal puede devenir en duelo complicado. Con el objetivo de desarrollar y probar un modelo explicativo del efecto de la resiliencia, el apoyo social y variables sociodeomográficas sobre la resolución del duelo perinatal, así como determinar los índices psicométricos de la Escala de Resiliencia Mexicana (RESIM) en mujeres con pérdida perinatal, se realizó un estudio transversal con 295 mujeres en duelo, quienes contestaron la Escala de Duelo Perinatal, la de Calidad de Red y la RESI-M. Los índices de confiabilidad y validez de la RESI-M resultaron satisfactorios. El modelo explicó $12 \%$ de la varianza del duelo, con índices de ajuste adecuados; dos factores resilientes fueron predictores significativos. El apoyo familiar y de amigos, la práctica religiosa y el número de hijos predijeron la resiliencia. Se discute el papel de estas variables en la previsión del duelo complicado y la utilidad de la RESI-M en la clínica e investigación.
\end{abstract}

Palabras clave: resiliencia, duelo perinatal, escalas, redes de apoyo, modelos estructurales

\begin{abstract}
Resilience and social support enable the possible solution of perinatal bereavement. With the objective to determine the psychometric indexes of the Mexican Scale of Resilience (RESI-M) in women with perinatal loss and to establish the relation between resilience and social support within bereavement in an explanatory model, a cross-sectional study with 295 Mexican women in mourning was performed; they answered the RESI-M, the Scale of Network Quality and the Scale of Perinatal Grief. The psychometric indexes of the RESI-M were satisfactory, and the AFC showed acceptable levels of adjustment. The model explained $12 \%$ of the variance of active grief, with adequate adjustment indices; two resilient factors were significant predictors; support from family and friends, religious practice, and number of children predicted resilience. The role of these variables in the anticipation of complicated grief and the usefulness of RESI-M in clinical and research are discussed.
\end{abstract}

Keywords: resilience, perinatal bereavement, scales, support networks, structural models

\footnotetext{
${ }^{1}$ Doctora en Psicología. Investigadora en Ciencias Médicas B. Instituto Nacional de Perinatología. Montes Urales 800, Lomas Virreyes CP.11000, Ciudad de México. Tel.: 55209900 ext. 147. Correo: motaceci@ hotmail.com

${ }^{2}$ Doctora en Psicología. Investigadora Titular B, Facultad de Psicología. Universidad Nacional Autónoma de México. Avenida Universidad 3000, CP. 04510, Ciudad Universitaria, Ciudad de México, México. Tel.: 5255 1126-6797. Correo: ncalleja@unam.mx

${ }_{3}^{3}$ Doctora en Psicología. Investigadora en Ciencias Médicas C. Instituto Nacional de Perinatología. Montes Urales 800, Lomas Virreyes CP.11000, Ciudad de México. Tel.: 55209900 ext. 147. Correo: clausanbra@yahoo.com

${ }^{4}$ Doctor en Psicología. Investigador en Ciencias Médicas. Instituto Nacional de Perinatología. Montes Urales 800, Lomas Virreyes CP.11000, Ciudad de México. Tel.: 55209900 ext. 147. Correo: locame2003@yahoo.com

${ }^{5}$ Pasante de la Licenciatura en Psicología. Facultad de Estudios Superiores Zaragoza, UNAM. Montes Urales 800, Lomas Virreyes CP.11000, Ciudad de México. Tel.: 55209900 ext. 147. Correo: jesbu@ gmail.com

Revista Iberoamericana de Diagnóstico y Evaluación - e Avaliação Psicológica. RIDEP · №58 · Vol.1 · 35-46 · 2021

ISSN: 1135-3848 print /2183-6051online
} 


\section{Introducción}

El término resiliencia en la psicología clínica se introdujo a partir de investigaciones sobre cambios de conducta en niños y jóvenes en riesgo de psicopatología por la exposición a circunstancias familiares adversas (Masten, 2007). Se ha definido como la capacidad de recuperarse, enfrentar desafíos y reorganizarse ante las dificultades (Wagnild \& Young, 1993; Wolin \& Wolin, 1993). Hu, Zhang y Wang (2015) se aproximan al concepto de resiliencia desde tres orientaciones: rasgo, que se refiere a una característica que ayuda a que el individuo afronte y se ajuste positivamente a la adversidad; resultado, que está en función del comportamiento que propicia la recuperación ante la adversidad, y proceso, relativo al mecanismo dinámico que favorece una rápida adaptación y recuperación. Luthar, Cicchetti y Becker (2000) afirman que la resiliencia es "un proceso dinámico que conlleva una adaptación positiva en contextos de gran adversidad" (p. 574); es decir, ser resiliente no es ser invulnerable a ésta, sino poder superarla adecuadamente, sin consecuencias negativas (García del Castillo, García del Castillo, López, \& Dias, 2016). Otros autores concuerdan en que es un patrón de pensamiento y conducta que se desarrolla cuando el individuo afronta eventos adversos que requieren una respuesta positiva para mantener o mejorar su calidad de vida (Bonanno, Westphal, \& Mancini, 2011; González-Arratia, Valdez, Oudhof, \& González, 2012).

\section{Resiliencia, apoyo social y duelo perinatal}

La muerte perinatal provoca intenso malestar emocional en los padres. El duelo perinatal involucra síntomas físicos y psicológicos que vulneran el aparato psíquico y rebasan las capacidades para afrontar y aceptar la pérdida, convirtiéndose en un trauma (Calderón \& Salazar, 2017).

La muerte de un ser querido activa un proceso dual de afrontamiento compuesto por dos mecanismos: uno orientado hacia la pérdida y su elaboración mediante expresión de emociones, añoranza, recuerdos y rumiación, y otro orientado hacia la restauración, generando estrategias para manejar los estresores surgidos (Strobe \& Schut,
1999). La oscilación entre ambos mecanismos resulta en un proceso de duelo normal (Strobe, Schute, \& Boerner, 2017). Se ha encontrado que el afrontamiento positivo (evaluación cognitiva o solución de problemas) se relaciona con la resiliencia y el crecimiento personal (García del Castillo et al., 2016). El afrontamiento puede entenderse como resultado de la resiliencia.

La pérdida perinatal puede provocar duelo complicado (Fernández, Pérez, Catena, Pérez, \& Cruz, 2016). Las dolientes experimentan culpa, vergüenza, arrepentimiento, miedo, estigma y deseos de morir (Davidson, 2018). Diversas investigaciones enfatizan los factores que intervienen en el desarrollo del duelo perinatal (Gold, Leon, Boggs, \& Sen, 2016; Mota, Calleja, Sánchez, \& Carreño, 2019; Peters, Lisy, Riitano, Jordan, \& Aromataris, 2015). Algunas de ellas destacan el papel de la resiliencia para adaptarse de manera efectiva (Kim \& Wilson, 2018; Leon, 2018). Yu et al. (2016) reportaron que la resiliencia y el miedo a la muerte predijeron los síntomas de duelo y su intensidad; las mujeres con patrones resilientes generaron respuestas adaptativas positivas frente a la adversidad, de tal forma que la resiliencia funcionó como un mecanismo autorregulador que las protegió de las consecuencias negativas de la muerte perinatal. Calderón y Salazar (2017) consideran que existen factores protectores y de riesgo para el duelo, que actúan dentro del proceso de resiliencia. Los protectores involucran el acompañamiento que la familia ofrece a la mujer en duelo, la percepción que ella tenga del suceso - de acuerdo con conceptos religiosos, espirituales y sociales -, las herramientas adquiridas en el desarrollo de su personalidad y el afrontamiento logrado durante la vida. Además, dado que la resiliencia es maleable, el desarrollo de intervenciones que la favorezcan en asesoramiento y en terapia, mejorará el ajuste al duelo (Bermejo, Magaña, Villacieros, Carabias, \& Serrano, 2012). A mayor capacidad de adaptación y aceptación de la pérdida, las actitudes resilientes en los padres se incrementarán, evitando el duelo complicado (Barreto, De la Torre, \& Pérez, 2012).

También se ha observado que tanto el apoyo familiar como el apoyo social percibido influyen en el proceso de duelo, amortiguando el impacto negativo de los acontecimientos estresantes 
(Mancini, Sinan, \& Bonano, 2015; Navea \& Tamayo, 2018)

El apoyo social ha sido conceptualizado como el conjunto de recursos sociales que una persona percibe tener disponibles (Gottlieb \& Bergen, 2010); es la experiencia que tienen los seres humanos de que son amados, cuidados, estimados y valorados como parte de una red social de asistencia mutua. El apoyo social puede ser proporcionado por la familia, los amigos, la pareja o, de manera formal, por grupos profesionales, instituciones o asociaciones (Melguizo, Hombrados, \& Martos, 2020).

En lo que respecta al duelo perinatal, McSpedden, Mullan, Sharpe, Breen y Lobb (2017) señalan que tanto el apoyo social incluyendo una sólida relación de pareja -, como las habilidades resilientes fungen como protectores para evitar su complicación. Sin embargo, brindar apoyo en el contexto de la pérdida es complejo y puede no amortiguar el estrés (Anusic \& Lucas, 2014). Es importante que las dolientes perciban que su red social es receptiva a escuchar sus preocupaciones e inquietudes para enfrentar mejor su pérdida (Navea \& Tamayo, 2018).

Según Martínez et al. (2019), el apoyo social y las habilidades resilientes son los principales recursos psicológicos que amortiguan el estrés y ofrecen otra perspectiva para enfrentar el duelo. Estudios recientes han mostrado que una mayor percepción de apoyo social se asocia significativamente con una disminución de las dificultades para elaborar el duelo y con mayor crecimiento personal (Oexle \& Sheehan, 2020).

\section{Medición de la resiliencia}

En psicología, se han hecho esfuerzos por medir la resiliencia. Wagnild y Young (1993) desarrollaron una escala basada en entrevistas con 24 mujeres de edad avanzada que se adaptaron con éxito a varias pérdidas propias de la vejez. Construyeron una escala de 25 ítems con dos factores: competencia personal y aceptación de la vida y de sí mismo, resultando altamente confiable y con evidencias de validez de constructo. Connor y Davidson (2003) construyeron la ConnorDavidson Resilience Scale, (CD-RISC), con 25 ítems tipo Likert, agrupados en cinco dimensiones; obtuvieron un alpha de Cronbach de .89 .
En Latinoamérica se han desarrollado algunos instrumentos. La Escala de Resiliencia SV-RES fue desarrollada en Chile por Saavedra y Villalta (2008), con 60 ítems divididos en doce factores (alfa =.96). En México se han construido varias escalas que miden este constructo, como la Escala de Resiliencia Materna (Roque, Acle, \& García, 2009), el Cuestionario de Resiliencia (fuerza y seguridad personal) de GonzálezArratia y Valdez (2012), con 32 ítems agrupados en tres dimensiones: factores protectores internos, externos y empatía (coeficiente de confiabilidad de.92), y la Escala de Resiliencia Mexicana (RESI-M) de Palomar y Gómez (2010), formada por 43 ítems y cinco factores que, de acuerdo con los autores, posee buenos índices de confiabilidad y validez $(\alpha=.93$ y $43.6 \%$ de varianza); fue validada en una muestra de jóvenes de ambos sexos.

El propósito del presente estudio fue evaluar el efecto tanto de las habilidades resilientes en mujeres que se encuentran en proceso de duelo por una muerte perinatal, como del apoyo social percibido y de variables sociodemográficas sobre el desarrollo del duelo en un modelo explicativo. Además, puesto que no se cuenta con una escala de resiliencia validada en esta población, un segundo objetivo fue evaluar las propiedades psicométricas de la RESI-M en mujeres en duelo perinatal.

\section{Método}

\section{Participantes}

En este estudio transversal, correlacional, participaron 295 mujeres mexicanas que habían sufrido pérdidas gestacionales y que acudían para su atención médica al Instituto Nacional de Perinatología de la Ciudad de México, un hospital de referencia del país, especializado en problemas de salud reproductiva. El muestreo fue no probabilístico intencional por cuota. Se incluyeron mujeres mayores de edad (de 18 a 44 años), con estudios mínimos de educación básica, independientemente de la patología reproductiva que presentaran; se consideró que el tiempo transcurrido desde la pérdida fuera de 10 años o menos, ya que el duelo, en los casos más graves, puede prolongarse durante muchos años 
Tabla 1. Características sociodemográficas y pérdidas perinatales de las participantes. $\mathrm{n}=295$

\begin{tabular}{|c|c|c|c|}
\hline \multicolumn{2}{|c|}{ Variables } & Rango & \multirow{2}{*}{$\frac{\text { Media (DE) }}{30.51(5.80)}$} \\
\hline \multirow{13}{*}{ Socio-demográficas } & Edad & $18-44$ años & \\
\hline & \multirow[t]{2}{*}{ Educación formal } & 6-20 años & $12.32(2.67)$ \\
\hline & & Categorías & Porcentaje \\
\hline & \multirow{3}{*}{ Estado civil } & Casada o en unión libre & 76.6 \\
\hline & & Solteras & 23.4 \\
\hline & & Sin hijos & 61.7 \\
\hline & \multirow{3}{*}{ Hijos } & 1 & 23.7 \\
\hline & & 2 & 9.5 \\
\hline & & 3 a 5 & 5.1 \\
\hline & \multirow[b]{2}{*}{ Empleo remunerado } & Con empleo & 30.2 \\
\hline & & Sin empleo & 69.8 \\
\hline & \multirow[b]{2}{*}{ Práctica religiosa } & Con práctica & 58.9 \\
\hline & & Sin práctica & 41.1 \\
\hline \multirow{12}{*}{ Pérdidas gestacionales } & \multirow{4}{*}{ Pérdidas gestacionales } & 1 & 56.9 \\
\hline & & 2 & 20.3 \\
\hline & & 3 & 14.9 \\
\hline & & 4 a 8 & 7.9 \\
\hline & \multirow{3}{*}{$\begin{array}{l}\text { Edad gestacional de la } \\
\text { última pérdida }\end{array}$} & Primer trimestre & 45.8 \\
\hline & & Segundo trimestre & 34.2 \\
\hline & & Tercer trimestre & 20.0 \\
\hline & \multirow{5}{*}{$\begin{array}{l}\text { Tiempo transcurrido } \\
\text { desde la última pérdida }\end{array}$} & $<1$ semana & 23.4 \\
\hline & & $>1$ semana a 1 mes & 10.2 \\
\hline & & $>1$ mes a 1 año & 21.0 \\
\hline & & $>1$ año a 5 años & 29.5 \\
\hline & & $>5$ años a 10 años & 15.9 \\
\hline
\end{tabular}

o incluso nunca resolverse. Se excluyó a mujeres que estuvieran embarazadas y fueron eliminadas del estudio quienes no contestaron todos los instrumentos. En la Tabla 1 se muestran las características sociodemográficas de la muestra.

\section{Instrumentos}

Cuestionario de datos sociodemográficos y gestacionales, desarrollado para este estudio, en el que se exploraron: edad, escolaridad, estado civil, práctica religiosa, número de embarazos, edad gestacional y tiempo de la pérdida.

Escala de Resiliencia Mexicana (RESI-M) de Palomar y Gómez (2010). Consta de 43 ítems agrupados en cinco factores: 1) Fortaleza y confianza en sí mismo, referido al esfuerzo por alcanzar metas, confianza de su éxito, fortaleza, optimismo y tenacidad para enfrentar sus retos; 2) Competencia social, midiendo la capacidad para relacionarse con los demás y la facilidad de hacer amigos disfrutando de la socialización; 3) Coherencia familiar, relacionada con la capacidad de establecer relaciones con la familia y recibir apoyo de ella, conservando la lealtad entre sus miembros; 4) Apoyo social, referido a contar con las personas en momentos difíciles, ayudar o ser capaz de dar aliento, y 5) Estructura personal, capacidad de organización y planeación de actividades, contar con reglas y actividades sistemáticas, aun en situaciones adversas. Estos factores explican el $43.6 \%$ de varianza tota. El alpha de Cronbach reportado por los autores es de 93. Con la finalidad de reducir los efectos de piso/techo, en el presente estudio se utilizaron seis opciones de respuesta (Totalmente en desacuerdo, Muy en desacuerdo, Desacuerdo, De acuerdo, Muy de acuerdo y Totalmente de acuerdo), en lugar de las cuatro de la escala original.

Escala de Duelo Perinatal de Potvin, Lasker y Toedter (1989), traducida y adaptada al español por Mota, Calleja, Aldana, Gómez y Sánchez (2011). Consta de 22 ítems agrupados en cuatro factores: duelo activo, depresión, culpa y aceptación. En la adaptación al español se obtuvo un alfa de Cronbach de .95 y una varianza total explicada de $78 \%$.

Escala de Calidad de Red (Nava, Reyes, \& Vega, 2001). Explora la percepción de ayuda emocional, instrumental y de comunicación que tienen las personas por parte de sus redes sociales; consta de cinco factores (apoyo de amigos, apoyo de la familia incluyendo a los padres, hermanos, 
pareja e hijos; falta de apoyo, apoyo religioso y apoyo de vecinos). Para este estudio se obtuvieron 20 reactivos agrupados en tres factores: apoyo de familia, apoyo de amigos y apoyo de pareja, los cuales explicaron el $71.3 \%$ de varianza total y un índice de confiabilidad $\alpha=.92$

\section{Procedimiento}

La aplicación se realizó en la consulta externa de la institución hospitalaria, de manera individual, en una sola sesión, por psicólogos entrenados. Con respecto a las consideraciones éticas, a las participantes se les explicaron las condiciones de su inclusión en el estudio, el objetivo, el carácter anónimo de su información y su participación voluntaria. Al aceptar su participación, firmaron un consentimiento informado y, al finalizar, se les ofreció atención psicológica.

Este trabajo forma parte de una investigación más amplia avalada por los comités de investigación y de ética del Instituto Nacional de Perinatología con número de protocolo: 2017-2-67.

\section{Análisis de los datos}

Los análisis estadísticos se efectuaron mediante el uso de los programas SPSS y AMOS, versión 22. El análisis psicométrico de la RESI-M se llevó a cabo discriminando los ítems mediante la obtención de la distribución de frecuencias (para detectar ítems con porcentaje $>50 \%$ en una de las opciones de respuesta), medidas descriptivas, sesgo y curtosis $(>2)$, correlaciones ítem-total (>.80) y diferencias entre grupos extremos con pruebas $t$ de Student $(p<.05)$. La estructura factorial de la escala se probó con análisis factorial confirmatorio (AFC); se utilizaron los índices de ajuste del modelo $\chi^{2} / g l$; AGFI; CFI y RMSEA. La consistencia interna se obtuvo con el índice alfa de Cronbach. Las relaciones entre las variables se calcularon con correlación de Pearson. Se efectuaron pruebas $t$ de Student y ANOVA para la comparación de los factores por las variables categóricas. Los predictores se determinaron con análisis de regresión lineal múltiple con el método introducir. Finalmente, el modelo derivado del análisis se probó con ecuaciones estructurales, considerando los índices de ajuste $\chi^{2}$; CFI, TLI, SRMR y RMSEA.

\section{Resultados}

\section{Análisis psicométrico de la RESI-M}

Para cada ítem se obtuvieron medidas descriptivas, distribución de frecuencias, índices de correlación ítem-total y discriminación entre grupos extremos. Con base en este análisis, y puesto que no discriminaron, se eliminaron siete de los 43 ítems de la escala: "Lo que me ha ocurrido en el pasado me hace sentir confianza para enfrentar nuevos retos", "Sé dónde buscar ayuda", "Me gustan los retos", "Mi futuro pinta bien", "Sé que puedo resolver mis problemas personales", "Cuando no estoy bien, sé que vendrán tiempos mejores" y "Me siento cómoda con otras personas".

Con los 36 reactivos restantes se realizó un AFC con el método de máxima verosimilitud; se evaluó la normalidad multivariada con la prueba de Mardia, la cual, de acuerdo con Bollen (1989), debe ser inferior a $\mathrm{p}(\mathrm{p}+2)$, donde $\mathrm{p}$ es el número de variables observadas. En este caso el índice obtenido fue 580.769 , inferior a $36(36+2)=1368$. Debido a sus bajas cargas factoriales o a sus altos valores residuales, fueron descartados los siguientes ítems: "Soy una persona fuerte", "Sé muy bien lo que quiero", "Sé cómo lograr mis objetivos", "Estoy satisfecha conmigo misma", "Me es fácil establecer contacto con nuevas personas", "Es fácil para mí tener un buen tema de conversación", "Fácilmente me adapto a situaciones nuevas", "Tengo una buena relación con mi familia", "Tengo amigos/familia que valoran mis habilidades", "Las reglas y la rutina, hacen de mi vida más fácil", "Mantengo mi rutina aún en momentos difíciles", y "Soy buena para organizar mi tiempo". Los 24 ítems finales tuvieron cargas significativas sobre sus respectivos factores, con $\lambda$ estandarizados oscilando entre $.91 \mathrm{y} .68$. El modelo presentó niveles adecuados de ajuste a los datos $\quad\left(\chi^{2} / g l=473.46 / 236=2.01 ; \quad\right.$ AGFI $=.86$; CFI=.95; RMSEA=.05; IC90\%： .065-.060). El factor 1 fue denominado: Claridad en los objetivos, en lugar de Fortaleza y confianza en sí mismo, ya que los ítems que lo componen reflejan la convicción y la claridad que las personas tienen en sus objetivos; los factores: Competencia social, Coherencia familiar, Apoyo social y Estructura personal conservaron sus denominaciones originales (ver Figura 1). 


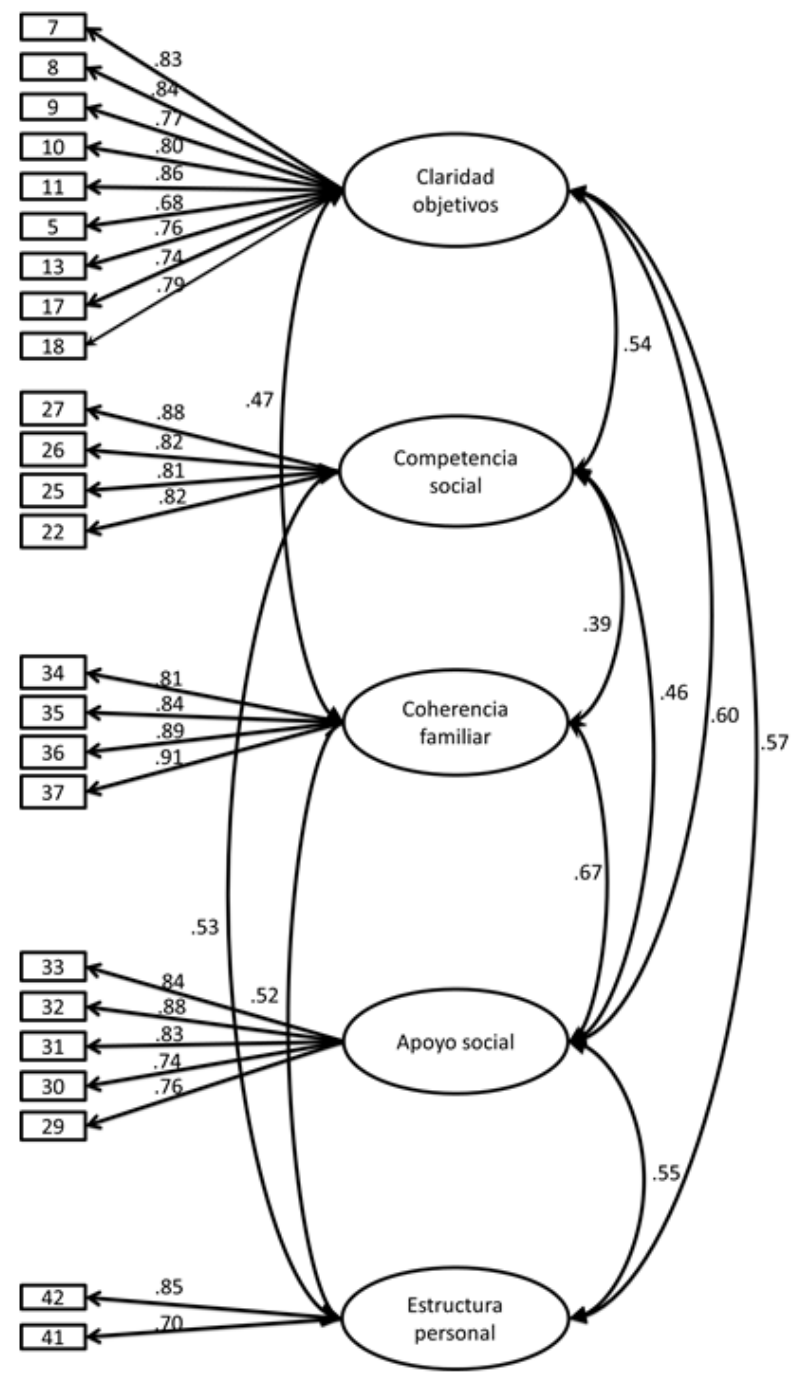

Figura 1. Estructura factorial de la RESI-M

La consistencia interna para la escala final de 24 ítems fue $\alpha=.944$. Los índices obtenidos para cada factor se muestran en la Tabla 2.

Las cinco subescalas correlacionaron positiva y significativamente entre sí (ver Tabla 2). Sus índices de correlación oscilaron entre $r=.368$ para Competencia social y Coherencia familiar, y $r=.608$ para Apoyo social y Coherencia familiar. Las medias de todas las subescalas estuvieron por arriba de la media teórica (3.5); la más alta fue Coherencia familiar $(M=5.01 \pm 0.92)$ y la más baja Competencia social $(M=4.06 \pm 1.01)$.

\section{Resiliencia y variables sociodemográficas}

Se probó el efecto de las variables sociodemográficas (edad, educación formal, estado civil, número de hijos, empleo remunerado y práctica religiosa) sobre los puntajes de resiliencia.
De ellas, el número de años escolares correlacionó positiva y significativamente con los cinco factores de resiliencia (Claridad de objetivos, $r=.208, p<.001$; Competencia social, $r=.155, p=.008$; Coherencia familiar, $r=.229$, $p<.001 ;$ Apoyo social, $r=.185, \quad p=.001, \quad \mathrm{y}$ Estructura personal, $r=.159, p=.006$ ); el número de hijos correlacionó positivamente con Claridad de objetivos ( $r=144, p=.013)$. Respecto de las variables categóricas, se encontraron diferencias significativas entre las participantes que contaban con un empleo remunerado y quienes no lo tenían en Claridad de objetivos, $t(293)=2.767, \quad p=.006 \quad(M=4.65, \quad D E=0.78$; $M=4.34, \quad D E=0.93, \quad$ respectivamente), y en Estructura personal, $\quad t(293)=2.432, \quad p=.016$ $(M=4.52, \quad D E=0.90 ; \quad M=4.23, \quad D E=0.97$, respectivamente). Las mujeres que practicaban una 
Tabla 2. Índices de correlación entre las subescalas de la RESI-M, medidas descriptivas e índices de consistencia interna

\begin{tabular}{lccccc}
\hline Subescalas & $\begin{array}{c}\text { Claridad de los } \\
\text { objetivos }\end{array}$ & $\begin{array}{c}\text { Competencia } \\
\text { social }\end{array}$ & $\begin{array}{c}\text { Coherencia } \\
\text { Familiar }\end{array}$ & $\begin{array}{c}\text { Apoyo } \\
\text { social }\end{array}$ & $\begin{array}{c}\text { Estructura } \\
\text { personal }\end{array}$ \\
\hline Claridad de los objetivos & 1 & & & & \\
Competencia social & $.494^{* *}$ & 1 & & & \\
Coherencia familiar & $.421^{* *}$ & $.368^{* *}$ & 1 & & \\
Apoyo social & $.538^{* *}$ & $.393^{* *}$ & $.608^{* *}$ & 1 & \\
Estructura personal & $.487^{* *}$ & $.455^{* *}$ & $.408^{* *}$ & $.430^{* *}$ & 1 \\
Media* & 4.45 & 4.06 & 5.01 & 4.70 & 4.33 \\
Desv. estándar & .89 & 1.01 & .92 & .97 & .97 \\
Coeficiente alfa de Cronbach & .94 & .90 & .93 & .91 & .74 \\
\hline
\end{tabular}

*Media teórica $=3.5$; rango de respuestas: 1 a $6 ; * * p<.01$.

Tabla 3. Índices de correlación entre los factores de Resiliencia y los de Apoyo social

\begin{tabular}{lccc}
\hline \multicolumn{1}{c}{ Resiliencia } & \multicolumn{3}{c}{ Apoyo social } \\
\cline { 2 - 4 } & Familia & Amigos & Pareja \\
\hline Claridad de objetivos & $.469^{* *}$ & $.370^{* *}$ & $.182^{*}$ \\
Competencia social & $.381^{* *}$ & $.512^{* *}$ & .099 \\
Coherencia familiar & $.600^{* *}$ & $.396^{* *}$ & $.212^{* *}$ \\
Apoyo social & $.830^{* *}$ & $.391^{* *}$ & $.301 * *$ \\
Estructura personal & $.389^{* *}$ & $.399^{* *}$ & $.235^{* *}$ \\
\cline { 2 - 3 }$*=.002: * p^{*} .001$. & &
\end{tabular}

religión se diferenciaron de quienes no la practicaban en Competencia social $t(283)=3.839$, $p<.001(M=4.25, \mathrm{DE}=1.04 ; M=3.80, D E=0.92$, respectivamente), y en Apoyo social, $t(283)=$ 2.455, $p=.015 \quad(M=4.82, \quad D E=0.94 ; \quad M=4.53$, $D E=0.97$, respectivamente).

\section{Resiliencia, pérdidas gestacionales y apoyo social}

De las variables relacionadas con la pérdida gestacional, sólo el número de pérdidas correlacionó negativamente con Coherencia familiar $(r=-.153, p=.008)$. Por otro lado, las correlaciones entre los factores de la RESI-M y los de la Escala de Calidad de Red resultaron positivas y altamente significativas, con excepción de la competencial social y el apoyo social de la pareja. Los índices más fuertes se obtuvieron entre el apoyo social familiar y los factores de resiliencia apoyo social y coherencia familiar (ver Tabla 3).

\section{Predictores de la resiliencia}

Se efectuaron regresiones lineales múltiples considerando como variables dependientes los factores de resiliencia y como predictores las variables que mostraron efecto sobre cada uno de ellos. En la Tabla cuatro, se presentan los índices obtenidos para cada modelo final, una vez que fueron excluidas las variables no significativas. Las pendientes de todos los predictores fueron positivos, con excepción del número de hijos, que se asoció negativamente con la claridad de objetivos. Sólo el apoyo social de la familia resultó significativo para todos los factores de resiliencia, particularmente en el de apoyo social, que explicó gran parte de su varianza; el apoyo de amigos estuvo presente en cuatro de ellos y el apoyo de la pareja sólo en el factor de estructura personal. Respecto de las características sociodemográficas, la variable años de estudio fue un predictor confiable para la coherencia familiar y el apoyo social, en tanto que el empleo remunerado lo fue para la claridad de objetivos y la estructura personal, mientras que la práctica religiosa lo fue para la competencia social.

\section{Resiliencia y duelo activo}

Al correlacionar las puntuaciones de duelo activo con los factores de resiliencia, tres de ellos resultaron negativos y significativos: Claridad de objetivos, $r=-.247, p<.001$; Competencia social, 
Tabla 4. Regresiones lineales múltiples para los factores de resiliencia

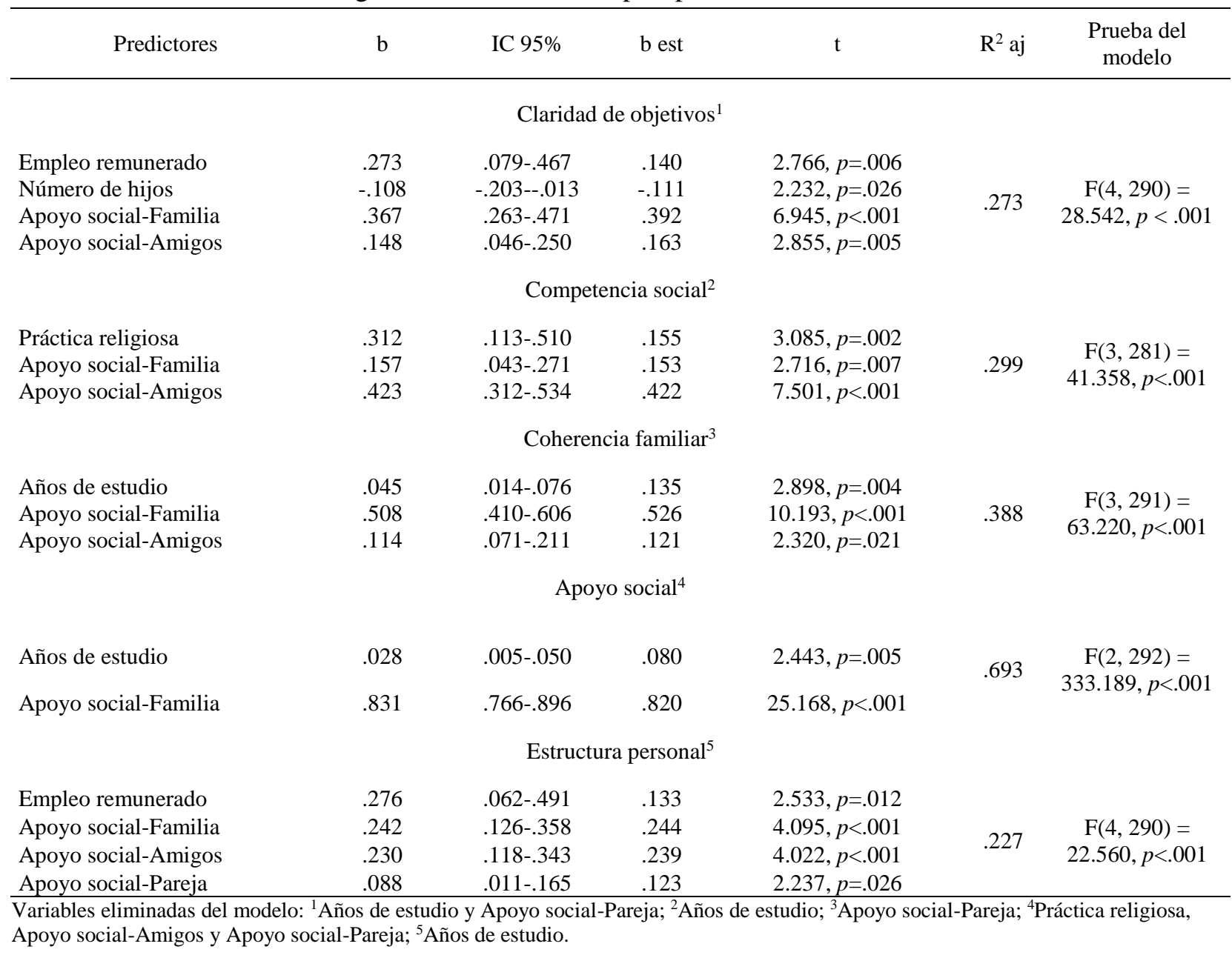

$r=-.249, \mathrm{p}<.001, \mathrm{y}$ Estructura personal, $r=-.160$, $p=.006$. Con ellos se efectuó una regresión lineal múltiple con el método introducir, encontrándose que sólo la Claridad de objetivos ( $b$ est.=-.163, $t=2.521, p=.012$ ) y la Competencia social ( $b$ est. $=-$ $.167, t=-2.573, p=.012)$ resultaron predictores confiables del Duelo activo. El modelo fue significativo, $F(2,292)=13.009, p<.001$ y explicó el $8.2 \%$ de la varianza.

\section{Apoyo social y duelo activo}

Las correlaciones de los factores de Apoyo social con Duelo activo fueron también negativas, pero bajas: Familia, $r=-.108, p=.063$; Amigos, $r=-$ $.180, p=.002$; Pareja, $r=.120, p=.040$. Al efectuar la regresión, se observó que únicamente el factor Amigos ( $b$ est.=-.180, $t=3.133, p=.002$ ) resultó predictor del Duelo activo, $F(1,293)=9.818$, $p=.002$ y explicó el $2.9 \%$ de su varianza.

\section{Modelo de resiliencia y duelo activo}

Con los resultados de las regresiones, se conformó un modelo explicativo incluyendo los dos factores de la resiliencia que predijeron el duelo activo (Claridad de objetivos y Competencia social). En el modelo se incorporaron los factores de apoyo social y las variables sociodemográficos que mostraron estar asociados con estos factores de resiliencia. Dicho modelo se sometió a un análisis de modelamiento estructural. Una vez realizadas las modificaciones requeridas, se obtuvieron índices de ajuste satisfactorios (ver Figura 2). El modelo explicó el $12.0 \%$ de la varianza de Duelo activo, siendo predictores significativos y en sentido inverso, los dos factores de resiliencia y el número de hijos (a mayor resiliencia y más hijos, menor duelo activo). En la explicación del $34.1 \%$ de la varianza de la Claridad de objetivos intervino fuertemente el Apoyo social de la Familia, además del número de hijos y tener un empleo remunerado. La varianza de la Competencia social fue explicada en un 27.4\%, principalmente por el Apoyo social de Amigos $\mathrm{y}$, en menor medida, por tener una práctica religiosa. La Competencia social tuvo efecto significativo sobre la Claridad de objetivos. 


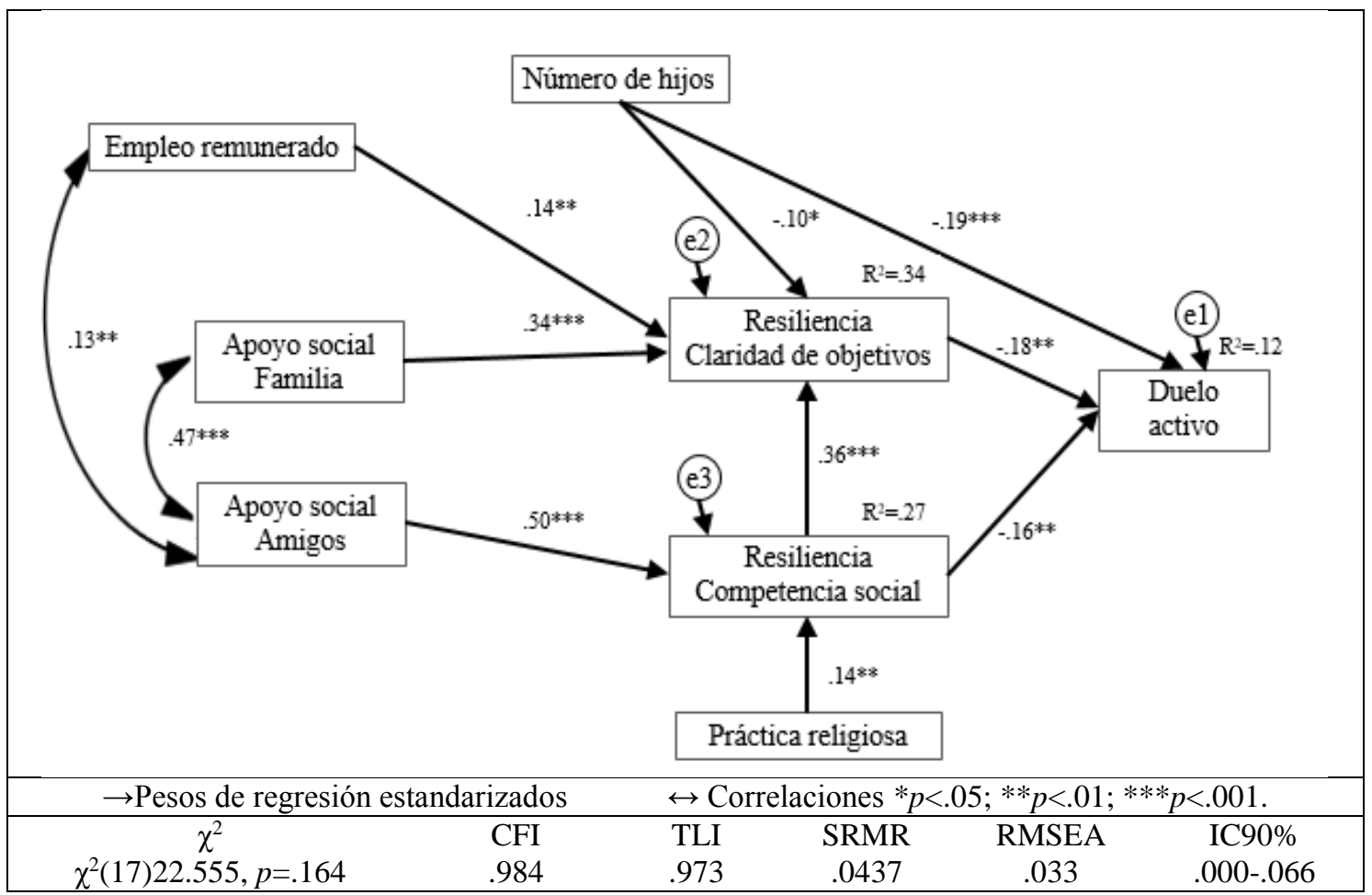

Figura 2. Modelo estructural de la relación Resiliencia - Duelo activo y las variables sociodemográficas y de apoyo social

\section{Discusión}

Ante la muerte perinatal se desencadena un proceso de duelo difícil de resolver, pues involucra la pérdida física, la del rol materno y las ilusiones, expectativas y proyectos que rodean al hijo. Como indica Fernández et al. (2016) es un evento traumático que puede desarrollar un duelo complicado, lo que implica la presencia de síntomas que provocan un deterioro de la salud mental y la minimización de la autonomía de la mujer en duelo (Calderón \& Salazar, 2017). No obstante, existen recursos psicológicos que sirven como protectores para el desarrollo del duelo normal, aun cuando se trate de un evento de gran impacto psicológico.

Uno de estos recursos es la capacidad resiliente, como se observó en los hallazgos del presente estudio, los cuales concuerdan con otras investigaciones en las que se destacael papel de la resiliencia en la aceptación de la muerte perinatal (Fernández et al., 2016; McSpedden et al., 2017).

Los resultados mostraron que las mujeres que tuvieron mayores habilidades resilientes, específicamente,claridad en objetivos de vida y competencia social, presentaron un duelo menos intenso frente a dicha pérdida. La presencia de otros hijos aumentó la posibilidad de desarrollar un duelo de menor intensidad; por el contrario, la ausencia de fortaleza y confianza en sí misma, aunado a la falta de claridad en los objetivos de vida, pareciera aumentar la posibilidad de que la muerte del hijo sea vivida como una situación extremadamente adversa, generadora de estrés y desesperanza. Como señalan Calderón y Salazar (2017), se vulnera el estado emocional a tal grado que se puede caer en un estado de vacío existencial, desarrollando depresión crónica. Otro hallazgo destaca que la claridad en los objetivos de vida y la competencia social se ven afectadas positivamente cuando las dolientes perciben el apoyo familiar, la presencia de otros hijos y/o cuando tienen una actividad laboral remunerada, pues dichas condiciones matizan los sentimientos (culpa, vergüenza y arrepentimiento), mencionados por Davidson (2018), así como los estigmas femeninos de sentirse incapaz e incompleta. La contención que brinda la red de apoyo de los grupos religiosos, así como el círculo de amistades, parecen favorecer las habilidades sociales necesarias para afrontar de una manera más efectiva la pérdida. Tal aseveración confirma lo encontrado por McSpedden et al. (2017) y Kim \& Wilson (2018), quienes mencionan que la resiliencia y un adecuado apoyo social, así como el componente religioso, son elementos 
protectores del duelo normal. En el presente estudio, el apoyo de pareja no tuvo ningún efecto sobre el duelo ni sobre la capacidad de resiliencia, quizá por el hecho de que las parejas también atraviesan un proceso de duelo similar.

El duelo por una muerte perinatal, como cualquier otro duelo, debe asumirse como parte del ciclo natural de la vida, para que se desencadene el comportamiento resiliente; es decir, las mujeres que presentaron duelos menos intensos, a pesar de estar en una situación altamente vulnerable, fueron capaces de mantener en niveles altos la claridad en sus objetivos, la competencia social, la coherencia familiar, la búsqueda de apoyo social y su estructura personal, lo que les permitió construir un significado a su pérdida y experimentar un crecimiento personal.

En concordancia con algunas investigaciones de otros países (v. gr., García del Castillo et al., 2016; McSpedden et al., 2017; Yu et al., 2016), puede destacarse que a pesar de que todas las mujeres experimentaron dolor por la pérdida, tristeza y pensamientos rumiativos, aquellas con características resilientes afrontaron dicha muerte de tal forma que pudieron seguir funcionando y construyendo significados en otras áreas de su vida. Las capacidades resilientes permiten que, durante el proceso de duelo perinatal, las mujeres cambien hábitos, pensamientos y respuestas que mantenían de manera progresiva a partir de percatarse de su embarazo hasta el momento de la pérdida.

En virtud de que en la investigación sobre duelo perinatal se requieren instrumentos sensibles y pertinentes para la medición de los constructos psicológicos relacionados, los resultados obtenidos en este estudio acerca de las características psicométricas de la RESI-M en mujeres que atraviesan un proceso de duelo perinatal, constituyen un valioso aporte. La escala conservó los cinco factores reportados en el instrumento original de Palomar y Gómez (2010) mostrando evidencias de confiabilidad y validez en la medición de la resiliencia en relación con el proceso de duelo perinata, aunque con menos ítems (24 versus 43), lo cual puede ser conveniente para su aplicación.

A pesar de que en el presente estudio se propone una versión corta del instrumento con mejores propiedades psicométricas que la original, como limitación del estudio queda pendiente evaluar si el instrumento acortado muestra las mismas propiedades psicométricas que la selección de los items realizada en el presente estudio, en ausencia de estos estudios se recomienda seguir aplicando el instrumento original aunque luego se analicen los ítems seleccionados.

Finalmente, el hecho de tener márgenes amplios en cuanto al tiempo transcurrido desde la pérdida, así como en la edad de las participantes, podría constituir una limitación para la medición de la intensidad del duelo, por lo que en investigaciones subsecuentes sería necesario controlar estas condiciones. Asimismo, un mayor tamaño de la muestra incrementaría la posibilidad de generalizar los resultados.

\section{Referencias}

Anusic, I., \& Lucas, R. E. (2014). Do social relationships buffer the effects of widowhood? A prospective study of adaptation to the loss of a spouse. Journal of Personality, 82, 367-378. https://doi.org/10.1111/jopy.12067

Barreto. P, De la Torre. O, Pérez-Marín, M. (2012) Detección de duelo complicado. Psicooncología, 3, 355-368. https://doi.org/10.5209/rev_PSIC.2013v9.n23.40902

Bermejo, J. C., Magaña, M., Villacieros, M., Carabias, R., \& Serrano, I. (2012). Estrategias de afrontamiento y resiliencia como factores mediadores de duelo complicado. Revista de Psicoterapia, 22(88), 85-95.

Bollen, K. A. (1989). Structural equations with latent variables. New York: John Wiley y Sons.

Bonanno, Westphal, \& Mancini (2011). Trauma and human resilience: Have we underestimated the human capacity to thrive after extremely aversive events? American $\begin{array}{lll}\text { Psychologist. } & \text { 59(1), 20-28. }\end{array}$ https://doi.org/10.1146/annurev-clinpsy032210-104526

Calderón, R., \& Salazar, P. (2017). La resiliencia un factor determinante en el proceso de duelo. Revista de Investigaciones Uniciencia. 3(1), 94-98 
Connor, K. M. \& Davidson, J. R. T. (2003). Development of a new resilience scale: The Connor-Davidson Resilience Scale (CDRISC). Depression and Anxiety. 18, 76-82. https://doi.org/10.1002/da.10113

Davidson, D. (2018). The need for bereavement support following perinatal loss. Bereavement Care, $\quad 37(1), \quad 31-34$. https://doi.org/1080/02682621.2018.1444316

Fernández, A. M., Pérez, M. M., Catena, M. A., Pérez, G. M., \& Cruz, Q. F. (2016). Influencia de la psicopatología emocional y el tipo de pérdida en la intensidad de los síntomas de duelo. Revista Iberoamericana de Psicología y Salud, 7(1), 15-24.

https://doi.org/10.1016/j.rips.2015.10.002

García del Castillo, R. J. A., García del Castillo L, A., López, S. C., \& Dias, P. C. (2016). Conceptualización teórica de la resiliencia psicosocial y su relación con la salud. Health and Addictions, 16(1), 59-68. http://dx.doi.org/10.21134/haaj.v16i1.263

Gold K. J., Leon I, Boggs M. E., \& Sen A. (2016). Depression and posttraumatic stress symptoms after perinatal loss in a populationbased sample. Journal of Women's Health. 25(3), 1-7.

https://doi.org/10.1089/jwh.2015.5284

González-Arratia, N. I., Valdez, J. L., Oudhof, H., \& González, S. (2012). Resiliencia y factores protectores en menores infractores y en situación de calle. Psicología y Salud, 22(1), 49-62. https://doi.org/10.25009/pys.v22i1.557

González-Arratia, N. I., \& Valdez, M. J. L. (2012). Análisis Factorial Confirmatorio del cuestionario de resiliencia en una muestra de niños. En Díaz Loving, R., Rivera A. S., y Reyes Lagunes, I. La Psicología Social en México (pp. 676-681). Vol. XIV. Eds. AMEPSO.

Gottlieb, B. H., Bergen, A. E. (2010). Social support concepts and measures. Journal of Psychosomatic Research, 69, 511-20. https://10.1016/j.jpsychores.2009.10.001

Hu, T., Zhang, D., \& Wang, J. (2015). A metaanalysis of the trait resilience and mental health. Personality and Individual Differences, 76, 18-27.

https://doi.org/10.1016/j.paid.2014.11.039
Kim, L., \& Wilson, E. E. (2018). Finding a way through: Integrating spirituality and sociocultural meaning in the face of infertility and perinatal loss. Socially Just Religious and Spiritual Interventions Ed. Springer, Cham. (pp. 87-97).

https://doi.org/10.1007/978-3-030-01986-08

Leon, I. G. (2018). Reproductive loss and its impact on the next pregnancy. In Motherhood in the Face of Trauma Ed. Springer, Cham. (pp. 69-81). https://doi.org/10.1007/978-3-319-65724-0_5

Luthar, S. S., Cicchetti, D., \& Becker, B. (2000). Research on resilience: Response to commentaries. Child Development, 71(3), 573-575.

https://doi.org/10.1111/1467-8624.00168

Mancini, A. D., Sinan, B., \& Bonanno, G. A. (2015). Predictors of prolonged grief, resilience, and recovery among bereaved spouses. Journal of Clinical Psychology, 71, 1245-1258. https://doi.org/10.1002/jclp.222

Mártínez, E. G., Fernández, C. E., González, M. E., Ávila, H. Y., Lorenzo, C. A., \& Vazquez, M. HL (2019) Apoyo social y resiliencia: Factores protectors en cuidadores principales de pacientes con hemodiálisis. Nefrológica, 22(2), 130-140.

https://doi.org/10.4321/S225428842019000200004

Masten, A. S. (2007). Resilience in developing systems: Progress and promise as the fourth wave rises. Development and Psychopathology, 19, 921-30 https://doi.org/10.1017/S0954570407000442

McSpedden, M., Mullan, B., Sharpe, L., Breen, L. J., \& Lobb, E. A. (2017). The presence and predictors of complicated grief symptoms in perinatally bereaved mothers from a bereavement support organization. Death Studies, 41(2), 112-117. doi: 10.1080/07481187.2016.1210696

Melguizo, G. A., Hombrados, M. I., \& Martos, M. M. J. (2020) La experiencia de un grupo de apoyo en el proceso de duelo de familiares de niños con cáncer. Un estudio cualitativo. Psicooncología, $\quad 17(1), \quad$ 117-129. https://doi.org/10.5209/psic.68245

Mota, G. C., Calleja, B. N., Aldana, C. E., Gómez, L. M. E., \& Sánchez, P. M. A. (2011). Escala 
de duelo perinatal: Validación en mujeres mexicanas con pérdida gestacional. Revista Latinoamericana de Psicología, 43(3), 419428.

Mota, G. C., Calleja, N., Sánchez, B. C., \& Carreño, M. J. (2019). Escala de Creencias sobre la Maternidad: Construcción y validación en mujeres mexicanas. Revista Iberoamericana de Diagnóstico y Evaluación - e Avaliação Psicológica, 1(50), 163-166. https://doi.org/10.21865/RIDEP50.1.13

Nava, Q. C., Reyes, L. I., \& Vega, V. Z. (2001). Estructura y calidad de red social en tres muestras familiares: Adolescentes, adultos jóvenes y maduros. Revista de Psicología Social y Personalidad, 17(1),17-34. Recuperado de https://www.medigraphic.com/pdfs/epsicologi a/epi-2014/epi143r.pdf

Navea, M. Tamayo, \& Hernández, A. (2018). Promoción de la resiliencia familiar en oncología pediátrica: Una revisión sistemática. Clínica Contemporánea, 9(3), 113. https://doi.org/10.5093/cc2018a18

Oexle, N., \& Sheehan, L. (2020) Apoyo social percibido y salud mental después de una pérdida por suicidio. Crisis: The Journal of Crisis Intervention and Suicide Prevention, 4l(1), 65-69. https://doi.org/10.1027/02275910/a000594

Palomar, L. J., \& Gómez, V. N. E. (2010). Desarrollo de una escala de medición de la resiliencia con mexicanos (RESI-M). Interdisciplinaria, 27(1), 7-22.

Peters, M. D. J., Lisy, K., Riitano, D., Jordan, Z., \& Aromataris, E. (2015). Caring for families experiencing stillbirth: Evidence-based guidance for maternity care providers. Women and Birth, 28(4), 272- 8. https://doi.org/10.1016/j.wombi.2015.07.003

Potvin, L., Lasker, J. N., \& Toedter, L. J. (1989). Measuring grief: A short version of Perinatal Grief Scale. Journal of Psychopathology and Behavioral Assessment, 11, 29-45. https://doi.org/10.1007/BF0096269

Roque, H. M. P., Acle, T. G., \& García, M. M. (2009). Escala de resiliencia materna: Un estudio de validación en una muestra de madres con niños especiales. Revista
Iberoamericana de Diagnóstico y Evaluación - e Avaliação Psicológica, 1(27), 107-132.

Saavedra, G. E. \& Villalta, P. M. (2008). Medición de las características resilientes: Un estudio comparativo en personas entre 15 y 65 años. Liberabit, 14(1), 31-40.

Strobe, M., Schut, H., \& Boerner, K. (2017). Cautioning health-care professionals: Bereaved persons are misguided through stages of grief. Omega, 74(4), 455-473. https://doi.org/10.1177/0030222817691870

Stroebe, M., \& Schut, H. (1999). The dual process model of coping with bereavement. Death Studies, 23, 197-224. https://doi.org/10.1080/074811899201046

Wagnild G., \& Young H. M. (1993). Development and psychometric evaluation of the resilience scale. Journal of Nursing Measurement, 1, 165-78. https://doi.org/10.1016/j.apnu.2010.05.001

Wolin, S., J., \&. Wolin, S (1993). The resilient self: How survivors of troubled families rise above adversity. Villard Books, New York

Yu, N. X., Chan, C. L., Zhang, J., \& Stewart, S. M. (2016). Resilience and vulnerability: Prolonged grief in the bereaved spouses of marital partners who died of AIDS. AIDS Care, 28(4), 441-444. https://doi.org/10.1080/09540121.2015.1112354 